\title{
ASYMPTOTIC THEORY OF LOW-DEGREE STELLAR ACOUSTIC OSCILLATIONS
}

\section{W. ROXBURGH}

Astronomy Unit, Queen Mary and Westfield College, London E1 4NS, UK

\section{S. V. VORONTSOV}

Institute of Astronomy, University of Cambridge, Cambridge CB3 0HA, UK, and Institute of Physics of the Earth, Moscow 123810, Russia (permanent address)

\begin{abstract}
We extend the second-order asymptotic description developed by Tassoul $(1980,1990)$ to the forth order, taking into account both gravity perturbations and realistic (non-polytropic) structure of the stellar envelope. We examine the accuracy of the asymptotic description by the direct computations for a solar model.
\end{abstract}

\section{THE ASYMPTOTIC DESCRIPTION}

The govering fourth-order system of equations of linear adiabatic oscillations can be reduced to the single second-order vector differential equation

$$
\frac{d^{2}}{d r^{2}}\left(\begin{array}{l}
\eta \\
P
\end{array}\right)+\mathbf{C}(r, \omega) \frac{d}{d r}\left(\begin{array}{l}
\eta \\
P
\end{array}\right)-\omega^{2} \mathrm{D}(r, \omega)\left(\begin{array}{l}
\eta \\
P
\end{array}\right)=0
$$

where $\eta(r)$ and $P(r)$ are proportional to the Eulerian pressure and gravitational potential pertirbations, and matrices $C(r, \omega)$ and $D(r, \omega)$ are determined by the equilibrium model (Vorontsov 1991). We derive the uniform asymptotic approximation to the solutions of the equation (1) using Bessel functions

$$
\left(\begin{array}{l}
\eta \\
P
\end{array}\right)=\left[\mathbf{Y}_{0}(r)+\frac{1}{\omega} \mathbf{Y}_{1}(r)+\frac{1}{\omega^{2}} \mathbf{Y}_{2}(r)+\frac{1}{\omega^{3}} \mathbf{Y}_{3}(r)+\ldots\right]\left(\begin{array}{c}
\omega^{1 / 2} J_{l+1 / 2}(\omega \varphi) \\
\omega^{1 / 2} j_{l+1 / 2}(\omega \varphi)
\end{array}\right)
$$

and determine matrices $Y_{i}(r)$ up to (and including) $Y_{3}(r)$. Near the surface, where asymptotic description becomes invalid, we match these solutions with exact frequency-dependent solutions. The resulted eigenfrequency equation is

$$
\begin{aligned}
\int_{0}^{R} \frac{d r}{c}+\frac{1}{\omega^{2}}\left[A_{0}+\ell(\ell+1) A_{\ell}\right] & +\frac{1}{\omega^{4}}\left[B_{0}+\ell(\ell+1) B_{\ell}+\ell^{2}(\ell+1)^{2} B_{\ell \ell}\right] \\
& \simeq \frac{\pi}{\omega}\left[n+\frac{\ell}{2}+\frac{1}{4}+\alpha(\omega)\right]
\end{aligned}
$$


where $\alpha(\omega)$ is frequency-dependent surface phase shift (Vorontsov 1991), and

$$
\begin{aligned}
A_{0} & =y(R)-\left[\frac{c}{2} \ln ^{\prime}\left(\frac{c}{h r^{2}}\right)+\frac{2}{3} \frac{c}{r}\right]_{r=R}, \quad A_{\ell}=-\frac{1}{2} \int_{0}^{R} c^{\prime} \frac{d r}{r}, \\
B_{0} & =\frac{1}{2} \int_{0}^{r_{m}}\left[-c y^{\prime 2}+\frac{c}{2} \ln ^{\prime}\left(\frac{c}{h r^{2}}\right)\left(N^{2}-4 \pi G \rho_{0}\right)^{\prime}\right] d r, \\
B_{\ell} & =\frac{1}{2} \int_{0}^{r}\left[c\left(c y^{\prime}+N^{2}\right)\right]^{\prime} \frac{d r}{r}-\frac{1}{2}\left[\frac{c}{r}\left(c y^{\prime}+N^{2}\right)\right]_{r=r_{m}}, \\
B_{\ell \ell} & =-\frac{1}{48} \int_{0}^{r m}\left(c^{3}\right)^{\prime \prime \prime} \frac{d r}{r}+\frac{1}{48}\left[\frac{\left(c^{3}\right)^{\prime \prime}}{r}+\frac{\left(c^{3}\right)^{\prime}}{r^{2}}+2 \frac{c^{3}}{r^{3}}\right]_{r=r_{m}}, \\
y(r) & =\frac{1}{2} \int_{0}^{r} c\left[\frac{c^{\prime \prime}}{2 c}-\left(\frac{c^{\prime}}{2 c}\right)^{2}-\frac{\ln ^{\prime \prime} h}{2}-\frac{\ln ^{\prime 2} h}{4}-\frac{\ln ^{\prime} h}{r}-\frac{N^{2}}{c^{2}}+\frac{4 \pi G \rho_{0}}{c^{2}}\right] d r, \\
h(r) & =\exp \int_{0}^{r}\left(\frac{N^{2}}{g}-\frac{g}{c^{2}}\right) d r .
\end{aligned}
$$

Constants $B_{0}, B_{\ell}, B_{\ell \ell}$ of the fourth-order term in the eigenfrequency equation (3) are independent on the position of the matching point $r_{m}$, if it is somewhere in the envelope, where second-order asymptotic is adequate (in local sense). For the small frequency separations $\nu_{n, \ell}-\nu_{n-1, \ell+2}=(2 \ell+3) D_{n, \ell}$, we get

$$
2 \pi \int_{0}^{R} \frac{d r}{c} \cdot D_{n, \ell} \simeq \frac{A_{\ell}}{\pi \nu}+\frac{1}{(2 \pi \nu)^{3}}\left[2 B_{\ell}+\left((2 \ell+3)^{2}+3\right) B_{\ell \ell}\right] .
$$

The accuracy of the asymptotic description of the absolute values of $p$-mode frequencies is demonstrated by Fig. 1. At higher frequencies $(\nu>4 \mathrm{mHz})$, significant improvement is clearly seen when the fourth-order description is compared with the second-order description. But there is no improvement at lower frequencies $(\nu<3 \mathrm{mHz})$, which indicates poor convergence of the asymptotic expansions

The accuracy of the description of $D_{n, \ell}$-values is illustrated by Fig. 2. The improvement is seen again at higher frequencies $(\nu>4 \mathrm{mHz})$ : the fourthorder approximation tends to describe reasonably the systematic difference between the exact values of $D_{n, 1}$ and $D_{n, 0}$. This difference is determined by the constant $B_{\ell \ell}$, with the kernel $\left(c^{3}\right)^{\prime \prime \prime} / r$ localized sharply in the solar core. Sufficiently accurate frequency measurements would allow one to utilise the high diagnostic capability of this difference.

The failure of the asymptotic descriptions at lower frequencies is due predominantly to the effects of gravity perturbations, which are large and difficult to treat accurately by the perturbational-type analysis. This conclusion is confirmed by additional computations in Cowling approximation.

We thank J. Christensen-Dalsgaard for his solar model used in the computations. S.V.V. thanks the UK Science \& Engineering Research Council, and "Zodiac" Scientific-Methodological Council for their partial support. 


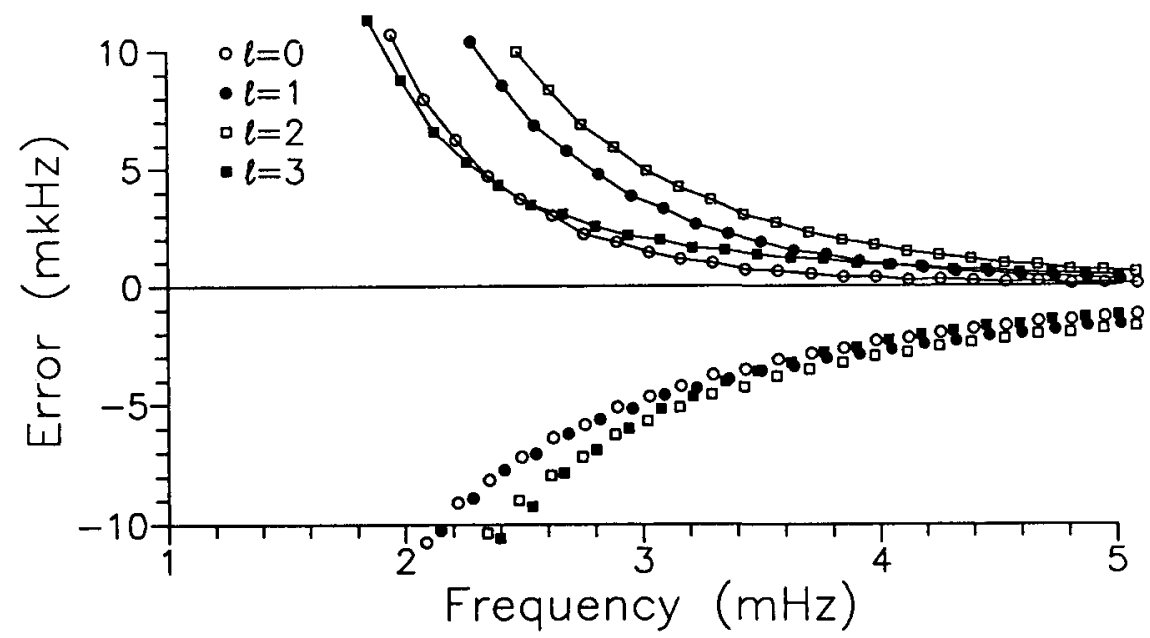

Fig. 1. Differences between asymptotic and exact eigenfrequencies, for the fourth-order (connected by solid lines) and for the second-order description. Computations were made for solar model 1 of Christensen-Dalsgaard (1982).

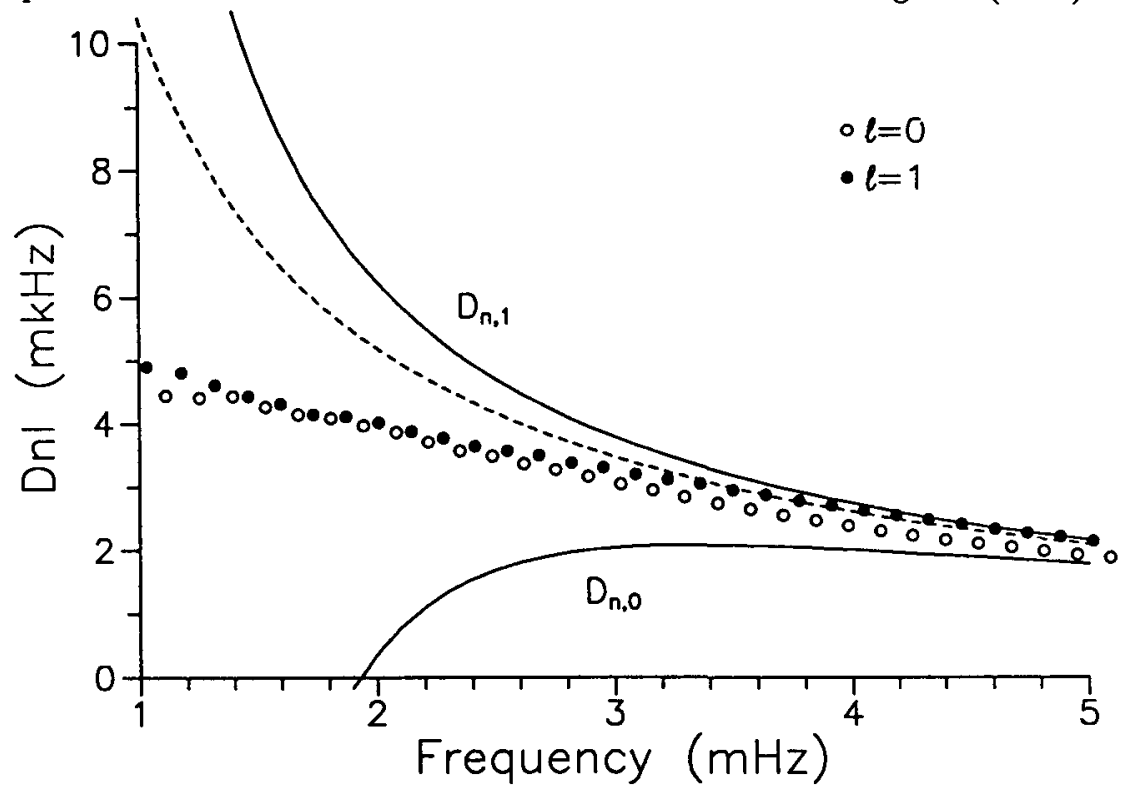

Fig. 2. Asymptotic values of $D_{n, \ell}$ resulted from the fourth-order (solid lines) and second-order (dashed line) asymptotic descriptions. The exact values of $D_{n, \ell}$ are shown by circules.

\section{REFERENCES}

Christensen-Dalsgaard, J. 1982, M.N.R.A.S., 199, 735.

Tassoul, M. 1980, Ap. J. Suppl., 43, 469.

Tassoul, M. 1990, Ap. J., 358, 313.

Vorontsov, S. V. 1991, Astron. Zh., 68, 808. 